\section{Abordagem das pesquisas em epidemiologia aplicada à gerontologia no Brasil: revisão da literatura em periódicos, entre 1995 e 2005}

\section{Approach to research in epidemiology applied to gerontology in Brazil: literature review of papers published between 1995 and 2005}

\section{Resumo}

O presente estudo teve como objetivo realizar uma revisão da literatura de forma exploratória e descritiva, buscando determinar a abordagem das pesquisas realizadas em epidemiologia aplicada à gerontologia no Brasil. Com esse intuito, foi efetuado um levantamento bibliográfico por meio da utilização do banco de dados da biblioteca virtual do SciELO, do LILACS e do PubMed. A busca dos artigos foi feita, exclusivamente, em periódicos incluídos nestes bancos de dados, sendo realizada a procura em artigos publicados no período compreendido entre os anos de 1995 a 2005. Os resultados encontrados demonstraram uma grande diversidade e abrangência nos enfoques das pesquisas realizadas, com o predomínio de pesquisas que abordam a área de psicologia, estudos delimitados por regiões e doenças relevantes para o envelhecimento. Houve também, um aumento de forma considerável no número total de pesquisas que foram realizadas a partir do ano de 2001, com aproximadamente $18,4 \%$ do total de artigos publicados encontrados somente no ano de 2005.

Palavras-chave: Epidemiologia. Gerontologia. Idosos.

Regina Bueno Ribas Pinto'

Laudelino Cordeiro Bastos ${ }^{2}$

'Mestranda em Tecnologia em Saúde, Pontifícia Universidade Católica do Paraná (PUC-PR)

${ }^{2}$ Professor do Mestrado em Tecnologia em Saúde, PUC-PR

Correspondência: Regina Bueno Ribas Pinto. Mestrado em Tecnologia em Saúde, Rua Imaculada Conceição, n 1155, Prado Velho, Curitiba, PR CEP 80215-901. E-mail: regifisio@hotmail.com 
Abstract

The present study had the objective of carrying out an exploratory and descriptive literature review, to determine the approach of research in epidemiology applied to gerontology in Brazil. The literature review used the databases of SciELO virtual library, LILACS and PubMed, and only included papers published in journals indexed these databases, in the period between 1995 and 2005. The results found were greatly diversified and comprehensive in terms of research focus with an important prevalence of issues related to psychology and conditions associated to aging. There was also an increase in the amount of research starting from 2001, with approximately $18.4 \%$ of the total papers published in 2005 .

Keywords: Epidemiology. Gerontology. Elderly.

\section{Introdução}

O crescimento da população de idosos, em números absolutos e relativos, é um fenômeno mundial relacionado com o aumento da expectativa de vida. No Brasil, segundo dados do Censo de 2000, as estimativas indicam que a população da terceira idade poderá exceder 30 milhões de pessoas para os próximos vinte anos, chegando a representar quase $13 \%$ da população total ${ }^{1}$. De acordo com dados da Pesquisa Nacional por Amostra de Domicílio (PNAD), publicados pelo Instituto Brasileiro de Geografia e Estatística (IBGE) em 1999, e realizado no período compreendido entre 1995 e 1999, ocorreu um aumento de 1,8 milhão no número total de idosos no país. O total de pessoas com 60 anos ou mais passou de $7,4 \%$ em 1989 para $8,3 \%$ em 1995, chegando a alcançar $9,1 \%$ no ano de $1999^{2}$.

O envelhecimento populacional e a expectativa de vida elevada acarretam no aumento significativo do número de idosos com doenças em relação à população em geral, principalmente as doenças de caráter crônico-degenerativo ${ }^{3}$. A pesquisa realizada por Filho e Ramos ${ }^{3}$ identificou que $61,4 \%$ dos idosos necessitaram procurar serviços de saúde nos últimos seis meses, sendo que $6,6 \%$ destes referiram pelo menos a ocorrência de um internamento. Outro dado importante é que $78,1 \%$ dos idosos apresentaram de uma a cinco doenças crônicas associadas. Confirmando estes achados, Ramos et $\mathrm{al}^{4}$ encontraram alta prevalência na ocorrência de doenças crônico-degenerativas nos idosos, além da presença de distúrbios psiquiátricos e alterações físicas freqüentes, referidas no estudo pela inabilidade destes idosos em realizar suas atividades da vida diária. De forma semelhante, Lebrão \& Laurenti ${ }^{5}$ identificaram uma limitação importante nas atividades dos idosos que apresentam doenças crônicas associadas, como, por exemplo, artrite, reumatismo e artrose.

Um dos fatores importantes na terceira idade são os episódios de depressão. São 
freqüentes, principalmente, em idosos octogenários, sendo o declínio da saúde considerado fator de risco para a instalação de quadros depressivos ${ }^{6}$. O estudo comparativo realizado por Porcu et $\mathrm{al}^{7} \mathrm{de}$ terminou uma prevalência elevada de sintomas depressivos nas populações estudadas. Tal amostra era constituída de idosos hospitalizados, institucionalizados e residentes na comunidade ${ }^{7}$.

Além da depressão, o transtorno de ansiedade generalizada foi apresentado no estudo de Xavier et $\mathrm{al}^{6}$ como um fator associado a sintoma depressivo e impacto negativo na qualidade de vida do idoso em relação à sua saúde ${ }^{8}$. Os transtornos depressivos constituem fatores que acarretam altos custos para os serviços de saúde, necessitando de cuidados altamente especializados para o tratamento e a intervenção destes idosos ${ }^{9}$.

Em relação a pesquisas com abordagem social, política e econômica, Veras ${ }^{10}$ identificou que, para atender as demandas relacionadas às condições de saúde e suporte social dos idosos, são necessários estudos diversos. A partir destes estudos, os gestores de saúde podem realizar mudanças nos modelos de assistência existentes atualmente.

Além da qualidade dos serviços prestados aos idosos, o fator relacionado aos custos é de fundamental importância, visto que, atualmente, os gastos com a saúde do idoso freqüentemente são elevados e onerosos ao país ${ }^{10}$. Em relação à qualidade, existe a necessidade de capacitar profissionais que estejam aptos e especializados para exercer suas funções frente aos aspectos multidisciplinares de tratamento e avaliação dos idosos ${ }^{11}$.

No Brasil, o aumento do número de idosos, provavelmente, motivou o interesse dos profissionais das diversas áreas em desenvolver pesquisas que abordem temas relacionados à epidemiologia. Esse interesse visa fundamentar a necessidade de cuidados aos idosos, também denominados anciãos, velhos ou grupo da terceira idade. Esses cuidados na área de saúde estão incluídos na geriatria, que é a parte da medicina responsável por tratar os problemas de saúde do idoso, e na gerontologia, que é a ciência que estuda o processo de envelhecimento do idoso sob múltiplos aspectos, entre os quais os biológicos, psicológicos, políticos, sociais, econômicos e espirituais ${ }^{12}$.

Além do conhecimento de geriatria e gerontologia, é importante identificar que a prática da epidemiologia apresenta três tipos de atividades analíticas: prestação de serviços, pesquisa e ensino em variados níveis. O tema epidemiologia da terceira idade encontra-se na subárea de epidemiologia $^{13}$. A partir dos dados do Diretório dos Grupos de Pesquisa, pode-se afirmar que existem 209 linhas de pesquisa referentes ao envelhecimento humano, divididas em 144 grupos de pesquisa no Brasil, o que sugere uma capacidade pequena de reprodução desta força de trabalho ${ }^{14}$.

Estudos epidemiológicos com abordagem gerontológica auxiliam, não só na compreensão do envelhecer, como também fornecem parâmetros para organizar e praticar políticas de saúde voltadas à população de idosos em geral.

Em vista do processo acelerado de envelhecimento populacional associado a comorbidades, o objetivo desta revisão da literatura foi identificar a abordagem das pesquisas em epidemiologia aplicada à gerontologia em periódicos no Brasil, durante um período de dez anos.

\section{Métodos}

Para se realizar este estudo, exploratório e descritivo, foi realizado um levantamento bibliográfico utilizando-se o banco de dados da biblioteca virtual do SciELO (http://www.scielo.br), o banco de dados LILACS, que está indexado na base de dados da Biblioteca Virtual em Saúde (BVS) da BIREME (http://www.bireme.br) e o banco de dados do PubMed (http:// www.pubmed.com). Nestas bases de dados foram identificados, exclusivamente, periódicos que estivessem indexados. O 
fato da utilização exclusiva de periódicos está relacionado com a avaliação realizada pela Coordenação de Aperfeiçoamento de Pessoal de Nível Superior (CAPES), que estabelece uma classificação, chamada de Qualis, para os periódicos que se encontram indexados nos bancos de dados das bibliotecas virtuais utilizadas.

Nesta pesquisa não foram incluídos livros, pois, embora haja uma forte e necessária reivindicação pela valorização destas publicações ${ }^{15}$, não há consenso sobre a avaliação que a própria CAPES efetua. Há estudos, como o de Raggio ${ }^{15}$, que questionam a avaliação atual da CAPES e propõe novos critérios de avaliação da produção acadêmica, incluindo a avaliação de livros.

Como critério de inclusão, foi definida a necessidade de os artigos no SciELO possuírem o período de publicação compreendido entre os anos de 1995 até 2005, e o termo epidemiologia, além dos termos disponíveis fornecidos, epidemiologia do envelhecimento e epidemiologia. Foi feita uma busca no formulário avançado, incluindo, além do termo epidemiologia, no campo de entrada assuntos, o termo idosos em todos os índices da base de dados, sendo encontrados 37 (trinta e sete) artigos no total. Com a troca do termo de todos os índices para geriatria e também gerontologia, foram encontrados os mesmos artigos que na busca para o termo idosos. Ao utilizar o termo envelhecimento em todos os índices, foram encontrados 15 (quinze) artigos, sendo 7 (sete) deles acrescidos aos achados anteriores, porém com a exclusão de 3 (três) por não se encaixarem nos critérios de inclusão do estudo.

Os artigos disponibilizados foram visualizados na íntegra e constam de periódicos publicados nas revistas a seguir: Revista Brasileira de Epidemiologia, Revista de Saúde Pública, Cadernos de Saúde Pública, Ciência e Saúde Coletiva, Revista Brasileira de Psiquiatria, Epidemiologia e Serviços de Saúde, Revista Brasileira de Medicina do Esporte, Revista de Psiquiatria Clínica, Revista da Sociedade Brasileira de Medicina Tropical, Pesquisa em
Odontologia Brasileira, Arquivos de Neuropsiquiatria, Revista Brasileira de Saúde Materno Infantil e Arquivo Brasileiro de Oftalmologia.

As buscas no LILACS e no PubMed tiveram como critérios de inclusão: período de publicação compreendido entre os anos de 1995 até 2005; as palavras selecionadas para busca deveriam estar contidas no título ou no resumo do artigo; os termos selecionados para a busca foram geriatria, idoso, envelhecimento, epidemiologia e gerontologia, de acordo com os descritores em saúde (DECS) estabelecidos pela BVS.

No LILACS foram encontrados três artigos com as características exigidas. Em relação à busca realizada no site do PubMed, foram selecionados os descritores correspondentes em inglês: epidemiology, aging, “old age”, aged, elderly and Brazil. O último descritor foi selecionado devido à pesquisa enfocar apenas periódicos com trabalhos realizados no Brasil. Foi encontrado um total de 11 (onze) artigos nessa base de dados, dos quais, após leitura detalhada, foram incluídos apenas 2 (dois) que atendiam aos critérios de inclusão.

Os artigos encontrados nos periódicos deveriam conter os termos relacionados acima, não necessariamente todos, porém o termo epidemiologia foi fator de inclusão neste artigo de revisão. A pesquisa deveria tratar de epidemiologia e estar relacionada com idosos, não sendo levado em conta o enfoque nem a área abordada.

De acordo com o $\mathrm{DECS}^{16}$, o termo geriatria é definido como ramo da medicina que estuda os aspectos fisiológicos e patológicos de idosos, inclusive alterações clínicas do envelhecimento e senilidade. Um termo sinônimo em português é gerontologia. $\mathrm{O}$ descritor idoso é definido como pessoa de 65 a 79 anos de idade, sendo o idoso acima de 80 anos ou mais considerado como octogenário, nonagenário e centenário. $\mathrm{O}$ descritor envelhecimento é definido como alterações que acontecem de forma gradual e irreversível na estrutura e no funcionamento de um organismo, e que ocorrem 
pela passagem do tempo. O descritor epidemiologia é definido como a ciência que estuda a distribuição de doenças e seus agravos nas comunidades, relacionando ambos com múltiplos fatores ${ }^{16}$.

No final das etapas de busca foram obtidos 41 (quarenta e um) artigos no SciELO, sendo que deste total foram utilizados para a análise 33 (trinta e três) deles que se encaixaram nos critérios pré-estabelecidos. No LILACS e no PubMed foram encontrados 14 (catorze) artigos, sendo utilizados 5 (cinco).

Com o material previamente definido, foram estabelecidas etapas para desenvolver a análise e posterior discussão. Primeiramente, foi realizada uma leitura de forma exploratória para definir o tema e o enfoque da pesquisa. Após os artigos serem selecionados, foram elaborados tabelas e gráfico para apresentar os resultados encontrados pela área e enfoque da pesquisa e também pelo ano de publicação do artigo. Após a criação das tabelas e do gráfico, foi realizada uma discussão para identificar a abordagem das pesquisas encontradas, a importância do direcionamento e o futuro para as pesquisas nas áreas de epidemiologia e gerontologia.

\section{Resultados e Discussão}

O tema epidemiologia associado à gerontologia foi identificado como de fun- damental importância para se estabelecer condições e determinantes de saúde dos idosos brasileiros, pois, com estes conhecimentos, será possível subsidiar políticas públicas e de saúde direcionadas a necessidades específicas ${ }^{17}$. Os artigos que tratam dos temas foram encontrados em 13 (treze) revistas no SciELo e 5 (cinco) no LILACS e no PubMed.

Os dados visualizados na Tabela 1 estão organizados pela abordagem da pesquisa, número de artigos encontrados, percentuais e o enfoque predominante. No item “outros”, estão incluídas as pesquisas que não se encaixaram nas abordagens identificadas.

Observa-se, por meio dos dados obtidos, uma grande variação dos temas de pesquisa que abordam a epidemiologia e estão associados ao tema gerontologia. As pesquisas com maior número de artigos, em relação aos demais, são as que enfocam depressão e os estudos regionais.

O tema depressão foi identificado, em estudos apresentados por Xavier et al. ${ }^{8} \mathrm{e}$ Snowdon ${ }^{9}$, como relevante e preocupante na população de idosos estudada, sendo encontrado um total de $21,1 \%$ de artigos que enfocam este tema. A epidemiologia, associada ao tema depressão no envelhecimento, encontra-se identificada com temas correlatos como ansiedade, capacidade cognitiva e sono nos idosos.

Com resultado igual $(21,1 \%)$, estão os

Tabela 1 - Distribuição percentual dos artigos encontrados pelas abordagens selecionadas e o enfoque predominante na pesquisa.

Table 1 - Percentage distribution of papers, divided by selected aprroaches and predominant focus of research.

\begin{tabular}{lccl}
\hline Abordagem da pesquisa & $\mathrm{N}^{\circ}$ de artigos & $\%$ & Enfoque predominante na pesquisa \\
\hline Biológica & 7 & 18,4 & Doenças \\
Social & 8 & 21,1 & Abordagem Regional (por Estado) \\
Nutricional & 2 & 5,2 & Obesidade e Sobrepeso \\
Psicológica & 8 & 21,1 & Depressão \\
Odontológica & 4 & 10,6 & Cárie \\
Mortalidade & 3 & 7,9 & Índices \\
Envelhecimento & 2 & 5,2 & Estudos epidemiológicos \\
Outros & 4 & 10,5 & Quedas, iatrogenia, entre outros \\
\hline Total & 38 & 100 & \\
\hline
\end{tabular}


artigos associados ao tema epidemiologia e as questões sociais. O enfoque inclui pesquisas por região (cidades e/ou estados do Brasil), com investigações em qualidade de vida e perfil epidemiológico, entre outros.

No âmbito regional, o Estado com maior número de pesquisas foi o de Minas Gerais, representando $37,5 \%$ do total de artigos. Em seguida, Pernambuco com 25\%, São Paulo com 25\% e Fortaleza com $12,5 \%$. A partir desses dados, observa-se uma tendência a se investigar as características destes idosos, para que a intervenção a ser realizada atue de maneira efetiva, de acordo com hábitos, crenças, clima, entre outros fatores regionais importantes.

Na proporção de 5,2\% do total estão as pesquisas com enfoque nutricional, sendo resultante de quantidades irrelevantes de artigos. Esta área necessita de maior número de estudos e aprofundamento nos temas relacionados com desnutrição e sobrepeso, os quais são abordados nas pesquisas encontradas ${ }^{18,19}$.

Com a mesma proporção $(5,2 \%)$ estão as pesquisas em estudos epidemiológicos, o que representa um valor insignificante pela importância do tema. O envelhecimento populacional é um desafio para a Saúde Pública atual, principalmente pelo fato de o Brasil ser um país em desenvolvimento e com desigualdades sociais aparentes $^{17}$. Isto justifica a relevância de estudos epidemiológicos de qualidade, que auxiliem no subsídio para a elaboração de políticas de saúde voltadas para os idosos, visando promover o envelhecimento saudável ${ }^{17}$

Os dados observados na Tabela 2 identificam que, no ano de 1995, existiam poucas pesquisas com o tema epidemiologia aplicada à gerontologia, o que persistiu até meados de 2000, com um total de publicações inexpressivo. O único artigo encontrado em 1995 aborda o tema relacionado com sintomas depressivos e déficit cognitivo em um grupo de idosos ${ }^{20}$.

Em geral, pode ser observado um crescente aumento nas publicações a partir do ano de 2001 (13,2\%), com um pico maior em 2005 (18,4\%). Este fenômeno é igualmente demonstrado no Gráfico 1, que apresenta essa relação de crescimento nas pesquisas com o tema em epidemiologia aplicada à gerontologia. Pressupõe-se, pelo conhecimento do envelhecimento populacional, fato amplamente divulgado em pesquisas como o Censo, que existe uma preocupação e uma necessidade elevada de dedicação por estudos nessa área. Este crescimento pronunciado no número de publicações, maior do que vinha acontecendo anteriormente no país, é um fato observado após a criação da Lei $n^{\circ} 10.741$ de $1^{\circ}$ de Outubro de $2003^{21}$ (Estatuto do Idoso), porém, este dado não apresenta correlação nenhuma, sendo apenas um ponto de vista. Esta lei foi uma forma encontrada para se estabelecer

Tabela 2 - Distribuição percentual dos artigos segundo o ano de publicação, período entre 1995 a 2005.

Table 2 - Percentage distribution of papers by year of publication, 1995 to 2005.

\begin{tabular}{lcc}
\hline Ano de Publicação & $\mathrm{N}^{\circ}$ de Artigos & \% de Artigos \\
\hline 1995 & 1 & 2,6 \\
1998 & 3 & 7,9 \\
1999 & 3 & 7,9 \\
2000 & 2 & 5,2 \\
2001 & 5 & 13,2 \\
2002 & 5 & 13,2 \\
2003 & 5 & 13,2 \\
2004 & 7 & 18,4 \\
2005 & 7 & 18,4 \\
\hline Total & 38 & 100 \\
\hline
\end{tabular}




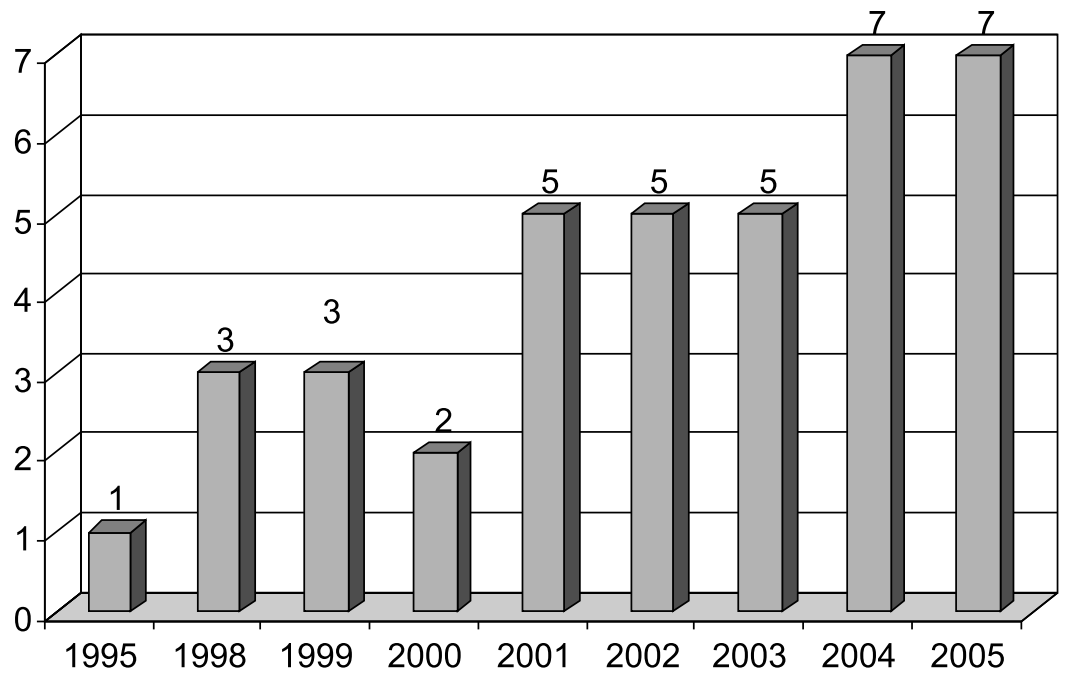

Gráfico 1 - Demonstra o aumento nas pesquisas com o passar dos anos, a partir do ano de 1995, com um aumento progressivo até 2005.

Graph 1 - Time trend in the number of papers, with a progressive increase from 1995 to 2005.

e regulamentar direitos dos idosos e entidades que os assistem, formalizando uma preocupação com aspectos da saúde e com a qualidade dos serviços prestados. Para estes aspectos, é relevante o enfoque de pesquisas com temas em epidemiologia aplicados à gerontologia, questões estas que visam identificar situações de intervenção nos idosos.

Embora o processo de envelhecimento traga consigo alterações importantes e múltiplas podem, porém, em muitos casos, serem modificadas por meio da identificação dos problemas e atuações diante de medidas preventivas ${ }^{22}$. Com esse intuito, as pesquisas brasileiras encontradas identificaram a importância da promoção da saúde do idoso e de seu bem estar, pelo desenvolvimento de projetos como o estudo Saúde, Bem estar e Envelhecimento (SABE) no município de São Paulo e o estudo Bambuí sobre saúde e envelhecimento (BHAS) no Brasil $^{5,23}$. Tais estudos apresentam experiências realizadas com grupos de idosos, visando principalmente identificar fatores que interferem na questão relacionada com qualidade de vida. O estudo $\mathrm{SABE}^{5}$ faz uma coleta de informações sobre as condições de vida dos idosos, definindo características da população estudada, como o estado de saúde, acesso e utilização de serviços e cuidados em saúde, além da identificação de dados sócio-econômicos. Outro estudo semelhante é o de Filho e Ramos ${ }^{3}$, que buscou identificar o perfil do idoso por nível socioeconômico, entre outros fatores. $\mathrm{O}$ que fica caracterizado é uma demanda aumentada de idosos na procura por serviços de saúde ${ }^{3}$, justificando a importância de pesquisas que forneçam subsídios para $o$ governo e profissionais da área de saúde. Já o estudo Bambuí ${ }^{23}$ visa identificar fatores que predizem eventos adversos à saúde dos idosos.

É importante identificar, nas pesquisas encontradas, que as características populacionais apresentadas são semelhantes às de outros estudos epidemiológicos realizados com idosos, o que salienta a importância da intervenção preventiva e de medidas de saúde pública específicas.

\section{Conclusão}

Durante o desenvolvimento desta revisão, foi observado que pesquisas epidemiológicas associadas a temas referentes aos idosos tiveram um crescimento acelerado nos últimos cinco anos. Conforme foi demonstrado, as pesquisas revelam que a 
correlação dos temas epidemiologia e gerontologia estabeleceram conhecimentos para diversas áreas, entre elas sociais, psicológicas, regionais e de saúde, com abordagens diversificadas.

As pesquisas identificam a necessidade emergente de se capacitarem recursos para a assistência dessa população específica, no âmbito da assistência a saúde e qualificação de profissionais, com a identificação de fatores de transição demográfica e epidemiológica populacional. É importante a implementação de serviços que promovam saúde, atuem na prevenção e no tratamento de doenças, com o objetivo de proporcionar incremento na qualidade de vida, buscando restabelecer a autonomia e manter a independência das pessoas durante o processo de envelhecimento.

Neste sentido, pesquisadores e instituições de ensino superior necessitam atuar e ampliar as linhas de pesquisa relaciona- das ao tema epidemiologia, principalmente associados à gerontologia. Este interesse pode alterar a representação do idoso na sociedade, direcionando pesquisas que enfoquem visões voltadas ao processo de envelhecimento e senescência e ao desenvolvimento de perfis do ponto de vista epidemiológico. Pode também possibilitar a ampliação do conhecimento sobre idoso, velhice e processo de envelhecimento fisiológico e curso de doenças.

Dessa forma, os profissionais que trabalham com idosos podem se utilizar das pesquisas para obter dados relevantes da atualidade, com o intuito de desenvolver planejamentos e decidir em prol da saúde do idoso. A divulgação de pesquisas e o desenvolvimento de ações voltadas ao conhecimento científico da epidemiologia voltada para a gerontologia auxiliam, ainda, na elaboração de ações adequadas para a transformação social, política e de saúde dos idosos brasileiros.

\section{Referências}

1. Fundação Instituto Brasileiro de Geografia e Estatística Diretoria de Pesquisas. Censos Demográficos. IBGE. Brasília; 2001. Disponível em http://www.ibge.gov.br. Acessado em 4 de maio de 2006.

2. Instituto Brasileiro de Geografia e Estatística (IBGE). Pesquisa Nacional por amostra de domicílios (PNAD). Rio de Janeiro; 1999. Disponível em http:// www.ibge.gov.br. Acessado em 5 de junho de2006.

3. Filho JMC, Ramos LR. Epidemiologia do envelhecimento no Nordeste do Brasil: resultados de inquérito domiciliar. Rev Saúde Pública 1999; 33(5): 445-53.

4. Ramos LR, Cendoroglo MS, Garcia JT, Najas MS, Perracini M, Paola CR, et al. Two-year follow-up study of elderly residents in S. Paulo, Brazil: methodology and preliminary results. Rev Saúde Pública 1998; 32(5): 397 407.

5. Lebrão ML, Laurenti R. Saúde, bem-estar e envelhecimento: o estudo SABE no Município de São Paulo. Rev Bras Epidemiol 2005; 8(2): 127-41.

6. Xavier FMF, Ferraz MPT, Bertollucci P, Poyares D, Moriguchi EH. Episódio depressivo maior, prevalência e impacto sobre qualidade de vida, sono e cognição em octogenários. Rev Bras Psiquiatr 2001; 23(2): 62-70.
7. Porcu M, Scantamburlo VM, Albrecht NR, Silva SP, Vallim FL, Araújo CR, et al. Estudo comparativo sobre a prevalência de sintomas depressivos em idosos hospitalizados, institucionalizados e residentes na comunidade. Acta Scientiarum 2002; 24(3): 713-17.

8. Xavier FMF, Ferraz MPT, Trenti CM, Argimon I, Bertolucci PH, Poyares D, et al. Transtorno de ansiedade generalizada em idosos com oitenta anos ou mais. Rev Saúde Pública 2001; 35(3): 294-302.

9. Snowdon J. How high is the prevalence of depression in old age? Rev Bras Psiquiatr 2002: 24(S1): 42-7.

10. Veras R. Em busca de uma assistência adequada à saúde do idoso: revisão da literatura e aplicação de um instrumento de detecção precoce e de previsibilidade de agravos. Cad Saúde Pública 2003; 19(3): 705-15.

11. Maia LC, Durante AMG, Ramos LR. Prevalência de transtornos mentais em área urbana no norte de Minas Gerais, Brasil. Rev Saúde Pública 2004; 38(5): 650-56.

12. Papaléo-Netto, M. Envelhecimento: desafio na transição do século. In: Papaléo-Netto M, Ponte JR, Duarte ALN, Ribeiro A, Cervado AM, Donato AF, et al. Gerontologia - a velhice e o envelhecimento em visão globalizada. São Paulo: Atheneu; 1996. p. 3-12. 
13. Guimarães R, Lourenço R, Cosac S. A pesquisa em epidemiologia no Brasil. Rev Saúde Pública 2001; 35(4): 321-40.

14. Prado SD, Sayd JD. A pesquisa sobre envelhecimento humano no Brasil: pesquisadores, temas e tendências. Ciência \& Saúde Coletiva 2004; 9 (3): 763-72.

15. Raggio LR. Avaliação de produtividade acadêmica: uma proposta de quantificação. Revista Brasileira de PósGraduação 2006; 3(6): 300-12.

16. Descritores em Ciências da Saúde. Disponível em http:// decs.bvs.br. Acessado em 18 de maio de 2006.

17. Lima-Costa MF, Barreto SM. Tipos de estudos epidemiológicos: conceitos básicos e aplicações na área do envelhecimento. Epidemiologia e Serviços de Saúde 2003; 12(4): 189-201.

18. Otero UB, Rozenfeld S, Gadelha AJ. Óbitos por desnutrição em idosos, São Paulo e Rio de Janeiro. Análise de séries temporais: 1980-1996. Rev Bras Epidemiol 2001; 4(3): 191-205.

19. Tavares EL, Anjos LA dos. Perfil antropométrico da população idosa brasileira. Resultados da Pesquisa Nacional sobre Saúde e Nutrição. Cad Saúde Pública 1999; 15(4): 759-68.
20. Silberman C, Souza C, Wilhems F, Kipper L, Wu V, Diogo $\mathrm{C}$ et. al. Cognitive deficit and depressive symptoms in a community group of elderly people: a preliminary study. Rev Saúde Pública 1995; 29(6): 444-50.

21. Brasil. Lei ${ }^{\circ} 10.741$ de 01 de Outubro de 2003. Dispõe sobre o Estatuto do idoso e dá outras providências. Diário Oficial da União, Brasília (3 de outubro de 2003).

22. Garrido R, Menezes PR. O Brasil está envelhecendo: boas e más notícias por uma perspectiva epidemiológica. Rev Bras Psiquiatr 2002; 24 (S1): 3-6.

23. Costa MFFL, Uchoa E, Guerra HL, Firmo JOA, Vidigal PG, Barreto SM. The Bambuí health and ageing study (BHAS): methodological approach and preliminary results of a population-based cohort study of the elderly in Brazil. Rev Saúde Pública 2000; 34(2): 126-35.

Recebido em: 22/08/06 Versão final reapresentada em: 16/07/07 Aprovado em: 27/07/07 